\title{
Projected Population-Wide Impact of Antiretroviral Therapy-Linked Isoniazid Preventive Therapy in a High-Burden Setting
}

August 16, 2018

\section{Section S1 Model Overview}

\section{Section S1.1 Compartments and Parameters}

\section{Compartment variable names:}

TB states are represented by a capital letter. Subscripts represent: stage of HIV infection and immunologic progression; then, after a decimal point, ART status if HIV infected; and finally, after a comma, TB drug-resistance phenotype if TB-infected.

Thus, $X_{h . a, d}(t)$ represents the number of individuals in TB state $X$, with HIV status $h$, ART status $a$ (omitted if HIV uninfected), and TB drug-resistance phenotype $d$ (omitted if no TB infection), at time $t$.

\section{Key Model Strata:}

- TB status:

- TB States:

* Uninfected

- not on IPT: $S$

- on IPT: $S_{I P T}$

- after IPT: $S_{I P T 2}$

* Latently infected

- not on IPT, infected recently (at risk for rapid progression): $L_{f}$

- not on IPT, infected remotely (at risk for delayed progression): $L_{s}$

· on IPT: $L f_{I P T}, L s_{I P T}$

- after IPT: $L f_{I P T 2}, L s_{I P T 2}$

* Active disease: $A$

- TB drug resistance states (subdivisions of all latently-infected and active-disease TB states):

* Drug-susceptible TB: $d=0$ (e.g. $\left.A_{h . a, 0}\right)$ for active drug-susceptible disease)

* MDR-TB (IPT does not prevent infection or reduce progression risk): $d=1$

- HIV and ART status:

* HIV-uninfected $(h=0)$ vs. infected $(h \in\{1,2,3\})$

* Immunologic status among HIV-infected: CD4>500 $(h=1), 500>\operatorname{CD} 4>200(h=2)$, or 200>CD4 $(h=3)$

* ART status among HIV infected: Not on ART (.1) or on ART (.2). 
Table S1: Parameter definitions

\begin{tabular}{|c|c|}
\hline Variable & Parameter description \\
\hline$\beta$ & Number of effective contacts for TB transmission per infectious person-year \\
\hline$\phi$ & Relative transmissibility of TB in people living with HIV (reflecting propensity to be smear negative, etc.) \\
\hline$\xi$ & Fraction of new TB infections that are MDR TB \\
\hline$\theta_{a}$ & Relative risk of TB progression and relative risk of HIV mortality, on vs off ART, for a given CD4 \\
\hline$\theta_{h 2}$ & Relative risk of TB progression, CD4 200-500 vs CD4 <200, for the same ART state \\
\hline$\theta_{h 1}$ & Relative risk of TB progression, CD $4>500$ vs CD $4<200$, for the same ART state \\
\hline$\theta_{i}$ & Relative risk of progression to active TB if latently infected, during IPT relative to never having received IPT \\
\hline$k_{\theta_{i}}$ & Proportion of reduction in progession risk that persists after IPT ends* \\
\hline$\sigma$ & 1/duration of "recently-infected" period with elevated risk for fast progression to active TB \\
\hline$\pi_{0}$ & Rate of rapid progression from latent to active TB after recent infection (HIV-) \\
\hline$\pi_{h 3}$ & Rate of rapid progression from latent to active TB after recent infection $(\mathrm{HIV}+, \mathrm{CD} 4<200 \text {, not on } \mathrm{ART})^{*}$ \\
\hline$\epsilon_{0}$ & Rate of delayed progression of latent TB to active (HIV-) \\
\hline$\epsilon_{h 3}$ & Rate of delayed progression of latent TB to active $(\mathrm{HIV}+, \mathrm{CD} 4<200$, not on ART)* \\
\hline$\tau_{d 0, h 0}$ & Rate of initiation of curative treatment for active drug-susceptible TB (HIV-) \\
\hline$\tau_{d 0, h 3}$ & Rate of initiation of curative treatment for active drug-susceptible TB (HIV,+ CD $4<200)^{* *}$ \\
\hline$\tau_{d 1}$ & Rate of effective treatment initiation for active MDR TB \\
\hline$\psi_{0}$ & Relative risk of reinfection, latently-infected versus uninfected, HIV-* \\
\hline$\mu$ & Background mortality rate \\
\hline$\mu_{t b, h 0}$ & TB-specific excess mortality rate (HIV-) \\
\hline$\mu_{t b, h 3}$ & TB-specific excess mortality rate $\left(\mathrm{HIV}+, \mathrm{CD} 4<200^{*}\right.$, not on ART) \\
\hline$\mu_{h 3}$ & HIV-specific excess mortality rate $(\mathrm{CD} 4<200)$ \\
\hline$\mu_{h 2}$ & HIV-specific excess mortality rate $(200<\mathrm{CD} 4<500)$ \\
\hline$\mu_{h 1}$ & HIV-specific excess mortality rate $(500<\mathrm{CD} 4)$ \\
\hline$\eta_{0}$ & Annual rate of new HIV infection \\
\hline$\eta_{1}$ & Rate of HIV progression from CD $>500$ to to CD4 200-500 \\
\hline$\eta_{2}$ & Rate of HIV progression from CD4 200-500 to CD4<200 \\
\hline$\eta_{3}$ & Rate of immunologic recovery on ART, from CD4 200-500 to CD4 >500) \\
\hline$\eta_{4}$ & Rate of immunologic recovery on ART, from CD4 <200 to CD4 200-500 \\
\hline$\chi_{1}$ & Net rate of ART initiation, HIV + with $500<\mathrm{CD} 4$, in absence of TB diagnosis \# \\
\hline$\chi_{2}$ & Net rate of ART initiation, HIV + with $200<\mathrm{CD} 4<500$, in absence of TB diagnosis \# \\
\hline$\chi_{3}$ & Net rate of ART initiation, HIV + with CD $4<200$, in absence of TB diagnosis\# \\
\hline$\kappa$ & Probability of IPT initation when starting ART \\
\hline$\kappa_{\text {onart }}$ & Rate of IPT initiation after already on ART \\
\hline$\rho$ & Proportion of 15 year olds with latent TB infection \\
\hline$\gamma$ & 1/Duration of IPT regimen (0 for continuous IPT) \\
\hline sens $s_{\text {xpert }}$ & Sensitivity of Xpert MTB/RIF screening for active TB (in ongoing or new ART recipients) \\
\hline ltf $u_{T B r x}$ & Proportion of individuals with active TB who do not receive TB treatment after TB diagnosis \\
\hline$l t f u_{a r t T B}$ & Proportion of individuals with active TB who do not initiate and remain on ART after TB diagnosis \\
\hline art $_{\text {screen }}$ & Indicator for whether ART initiators are screened for Active TB \\
\hline ipt $_{\text {screen }}$ & Indicator for whether IPT initiators are screened for Active TB \\
\hline$i n f_{d}$ & Indicator for whether infection with a given TB strain can occur while on IPT \\
\hline $\begin{array}{l}\text { *The relativ } \\
{ }^{*} \text { Thus, } \pi_{h H} \\
\tau_{d 0, h 1}=\tau_{d 0}\end{array}$ & $\begin{array}{l}\text { sk of progression to active TB after IPT ends, relative to never having received IPT, is } \theta_{i}^{\prime}=1-k_{\theta_{i}}\left(1-\theta_{i}\right) \text {. } \\
\theta_{h H} \pi_{h 3} \text { and } \epsilon_{h H}=\theta_{h H} \epsilon_{h 3} \text { for } H \in 1,2 \text {. } \\
=\left(\tau_{d 0, h 0}+\tau_{d 0, h 3}\right) / 2 ; \text { and } \mu_{t b, h 1}=\mu_{t b, h 2}=\left(\mu_{t b, h 0}+\mu_{t b, h 3}\right) / 2 \text {, assumed. } \\
1=\psi_{h 2}=1-\left(1-\psi_{0}\right) / 2 \text {, assumed. } \\
\text { ount for non-adherence. }\end{array}$ \\
\hline
\end{tabular}




\section{Section S1.2 Model Equations}

Force of infection: Drug-susceptible force of infection is proportional to prevalence, modified by HIV status. A parameter $\xi$ determines the fraction of new infections that are MDR.

$$
\begin{aligned}
\lambda_{0}(t) & =\beta \frac{\left(A_{0,0}(t)+\phi \sum_{i \neq 0} A_{i, 0}(t)\right)}{N} \\
\lambda_{1}(t) & =\lambda_{0}(t) \frac{\xi}{1-\xi} \\
\lambda(t) & =\lambda_{0}(t)+\lambda_{1}(t)
\end{aligned}
$$

Notes on Representation of MDR TB: The main purpose of including MDR TB in this model is to represent a fraction of the population with LTBI for whom preventive therapy will be ineffective (recognizing that IPT in particular may have some impact on some MDR strains with inhA mutations, and may also be ineffective in some INH-monoresistant LTBI). We use a simple representation of MDR TB as a parallel epidemic. MDR comprises a defined fraction of new TB infections, and has a longer time to effective treatment than DS TB but the same natural history otherwise. These parameters are calibrated to the observed local prevalence of MDR among TB cases.

An additional quantity to define for later use: Proportion of active TB cases that are detected and treated for TB when starting ART:

$$
\text { detected }=\left(\operatorname{art}_{\text {screen }}+\kappa\left(1-\operatorname{art}_{\text {screen }}\right) i p t_{\text {screen }}\right) * \text { sens }_{\text {xpert }} *\left(1-\text { ltf } u_{T B r x}\right)
$$

TB-Susceptible Compartments $(S)$ : TB-susceptible populations can be exposed to infectious persons and become infected with a hazard of $\lambda$ (the force of infection). In addition, they can transition between HIV states (serostatus, CD4, ART status). In addition, they experience a risk of dying based on background and HIV-related mortality.

$$
\begin{gathered}
S_{h=0}^{\prime}=-\left(\eta_{0}+\mu+\lambda\right) S_{h=0}+(1-\rho) \sum \text { deaths } \\
S_{h=1.1}^{\prime}=\eta_{0} S_{h=0}-\left(\eta_{1}+\mu+\mu_{h 1}+\lambda+\chi_{1}\right) S_{h=1.1} \\
S_{h=2.1}^{\prime}=\eta_{1} S_{h=1.1}-\left(\eta_{2}+\mu+\mu_{h 2}+\lambda+\chi_{2}\right) S_{h=2.1} \\
S_{h=3.1}^{\prime}=\eta_{2} S_{h=2.1}-\left(\mu+\mu_{h 3}+\lambda+\chi_{3}\right) S_{h=3.1} \\
S_{h=1.2}^{\prime}=(1-\kappa) \chi_{1} S_{h=1.1}+\eta_{3} S_{h=2.2}-\left(\mu+\theta_{a} \mu_{h 1}+\lambda+\kappa_{\text {onart }}\right) S_{h=1.2} \\
S_{h=2.2}^{\prime}=(1-\kappa) \chi_{2} S_{h=2.1}+\eta_{4} S_{h=3.2}-\left(\mu+\theta_{a} \mu_{h 2}+\lambda+\kappa_{a}+\eta_{3}\right) S_{h=2.2} \\
S_{h=3.2}^{\prime}=(1-\kappa) \chi_{3} S_{h=3.1}-\left(\mu+\theta_{a} \mu_{h 3}+\lambda+\kappa_{a}+\eta_{4}\right) S_{h=3.2} \\
S_{I P T, h=1.2}^{\prime}=\kappa_{a} S_{h=1.2}+\kappa \chi_{1} S_{h=1.1}+\eta_{3} S_{I P T, h=2.2}-\left(\gamma+\mu+\theta_{a} \mu_{h 1}+\lambda_{0} i n f_{d}+\lambda_{1}\right) S_{I P T, h=1.2} \\
S_{I P T, h=2.2}^{\prime}=\kappa_{a} S_{h=2.2}+\kappa \chi_{2} S_{h=2.1}+\eta_{4} S_{I P T, h=3.2}-\left(\gamma+\mu+\theta_{a} \mu_{h 2}+\lambda_{0} i n f_{d}+\lambda_{1}+\eta_{3}\right) S_{I P T, h=2.2} \\
S_{I P T, h=3.2}^{\prime}=\kappa_{a} S_{h=3.2}+\kappa \chi_{3} S_{h=3.1}-\left(\gamma+\mu+\theta_{a} \mu_{h 3}+\lambda_{0} i n f_{d}+\lambda_{1}+\eta_{4}\right) S_{I P T, h=3.2} \\
S_{I P T 2, h=1.2}^{\prime}=\gamma S_{I P T, h=1.2}+\eta_{3} S_{I P T 2, h=2.2}-\left(\mu+\theta_{a} \mu_{h 1}+\lambda\right) S_{I P T 2, h=1.2} \\
S_{I P T 2, h=2.2}^{\prime}=\gamma S_{I P T, h=2.2}+\eta_{4} S_{I P T 2, h=3.2}-\left(\mu+\theta_{a} \mu_{h 2}+\lambda+\eta_{3}\right) S_{I P T 2, h=2.2} \\
S_{I P T 2, h=3.2}^{\prime}=\gamma S_{I P T, h=3.2}-\left(\mu+\theta_{a} \mu_{h 3}+\lambda+\eta_{4}\right) S_{I P T 2, h=3.2}
\end{gathered}
$$




\section{Recently-Infected Latent Compartments $(L f)$ :}

Populations may become infected at a state-dependent rate $\lambda$, reduced by $\psi$ if previously latently infected. Rates of progression to active disease are higher $(\epsilon$ versus $\pi$ ) during recent infection, depend on HIV state, and may be reduced by $\mathrm{ART}$ and/or by current or prior IPT. The rate of flow $\sigma$ into the remotely-infected compartments is reduced by reinfections, expressed as an exponential term.

Not on IPT:

$$
\begin{aligned}
L f_{h=0, d=D}^{\prime}= & \lambda_{D}\left(S_{h=0}+\psi_{0}\left(L f_{h=0, d \neq D}+\sum_{d} L s_{h=0, d}\right)\right) \\
& -\left(\eta_{D}+\mu+\pi_{0}+\sigma \exp \left(-\psi_{0} \lambda_{D} / \sigma\right)+\psi_{0} \lambda_{d \neq D}\right) L f_{h=0, d=D} \\
L f_{h=H .1, d=D}^{\prime}= & \lambda_{D}\left(S_{h=H .1}+\psi_{H}\left(L f_{h=H .1, d \neq D}+\sum_{d} L s_{h=H .1, d}\right)\right)+\eta_{(H-1)} L f_{h=(H-1) .1, d=D} \\
& -\left(\mu+\mu_{h H}+\eta_{H \mid H \in(1,2)}+\chi_{H}+\pi_{h H}+\sigma \exp \left(-\psi_{H} \lambda_{D} / \sigma\right)+\psi_{H} \lambda_{d \neq D}\right) L f_{h=H .1, d=D} \\
L f_{h=H .2, d=D}^{\prime}= & \left.\lambda_{D}\left(S_{h=H .2}+\psi_{H} L f_{h=H .2, d \neq D}+\sum_{d}\left(\psi_{H} L s_{h=H .2, d}+S_{I P T 2, h=H .2}+\psi_{H} L f_{I P T 2, h=H .2, d}+\psi_{H} L s_{I P T 2, h=H .2, d}\right)\right)\right) \\
& +(1-\kappa)\left(\chi_{H} L f_{h=H .1, d=D}\right)+\eta_{(H+2) \mid H \in(1,2)} L f_{h=(H+1) .2, d=D} \\
& -\left(\mu+\theta_{a} \mu_{h H}+\eta_{(H+1) \mid H \in(2,3)}+\theta_{a} \pi_{h H}+\sigma \exp \left(-\psi_{H} \lambda_{D} / \sigma\right)+\psi_{H} \lambda_{d \neq D}+\kappa_{a}\right) L f_{h=H .2, d=D}
\end{aligned}
$$

Recently-Infected Latent, on-IPT Compartments:

$$
\begin{aligned}
L f_{I P T, h=1.2, d=0}^{\prime}= & \kappa_{a} L f_{h=1.2, d=0}+\kappa\left(\chi_{1} L f_{h=1.1, d=0}\right)+\eta_{3} L f_{I P T, h=2.2, d=0} \\
& +\lambda_{0} i n f_{0} \sum_{d}\left(S_{I P T, h=1.2, d}+\psi_{1} L s_{I P T, h=1.2, d}\right) \\
& -\left(\gamma+\mu+\theta_{a} \mu_{h 1}+\theta_{a} \theta_{i} \pi_{h 1}+\sigma \exp \left(-\psi_{1} \lambda_{0} i n f_{0} / \sigma\right)+\psi_{1} \lambda_{0} i n f_{0}+\psi_{1} \lambda_{1}\right) L f_{I P T, h=1.2, d=0} \\
L f_{I P T, h=1.2, d=1}^{\prime}= & \kappa_{a} L f_{h=1.2, d=1}+\kappa\left(\chi_{1} L f_{h=1.1, d=1}\right)+\eta_{3} L f_{I P T, h=2.2, d=1} \\
& +\lambda_{1} \sum_{d}\left(S_{I P T, h=1.2, d}+\psi_{1} L s_{I P T, h=1.2, d}\right) \\
& -\left(\gamma+\mu+\theta_{a} \mu_{h 1}+\theta_{a} \pi_{h 1}+\sigma \exp \left(-\psi_{1} \lambda_{0} / \sigma\right)+\psi_{1} \lambda_{0} i n f_{0}+\psi_{1} \lambda_{1}\right) L f_{I P T, h=1.2, d=1} \\
L f_{I P T, h=2.2, d=0}^{\prime}= & \kappa_{a} L f_{h=2.2, d=0}+\kappa\left(\chi_{2} L f_{h=2.1, d=0}+\eta_{4} L f_{I P T, h=3.2, d=0}\right. \\
& +\lambda_{0} i n f_{0} \sum_{d}\left(S_{I P T, h=2.2, d}+\psi_{2} L s_{I P T, h=2.2, d}\right) \\
& -\left(\gamma+\mu+\theta_{a} \mu_{h 2}+\eta_{3}+\theta_{a} \theta_{i} \pi_{h 2}+\sigma \exp \left(-\psi_{2} \lambda_{0} i n f_{0} / \sigma\right)+\psi_{2} \lambda_{0} i n f_{0}+\psi_{2} \lambda_{1}\right) L f_{I P T, h=2.2, d=0} \\
L f_{I P T, h=2.2, d=1}^{\prime}= & \kappa_{a} L f_{h=2.2, d=1}+\kappa\left(\chi_{2} L f_{h=2.1, d=1}+\eta_{4} L f_{I P T, h=3.2, d=1}\right. \\
& +\lambda_{1} \sum_{d}\left(S_{I P T, h=2.2, d}+\psi_{2} L s_{I P T, h=2.2, d}\right) \\
& -\left(\gamma+\mu+\theta_{a} \mu_{h 2}+\eta_{3}+\theta_{a} \pi_{h 2}+\sigma \exp \left(-\psi_{2} \lambda_{0} / \sigma\right)+\psi_{2} \lambda_{0} i n f_{0}+\psi_{2} \lambda_{1}\right) L f_{I P T, h=2.2, d=1} \\
= & \kappa_{a} L f_{h=3.2, d=0}+\kappa\left(\chi_{3} L f_{h=3.1, d=0}\right. \\
& +\lambda_{0} i n f_{0} \sum_{d}\left(S_{I P T, h=3.2, d}+L s_{I P T, h=3.2, d}\right) \\
& -\left(\gamma+\mu+\theta_{a} \mu_{h 3}+\eta_{4}+\theta_{a} \theta_{i} \pi_{h 3}+\sigma \exp \left(-\lambda_{0} i n f_{0} / \sigma\right)+\lambda_{0} i n f_{0}+\lambda_{1}\right) L f_{I P T, h=3.2, d=0} \\
L f_{I P T, h=3.2, d=0}^{\prime} & -\left(\gamma+\mu+\theta_{a} \mu_{h 3}+\eta_{4}+\theta_{a} \pi_{h 3}+\sigma \exp \left(-\lambda_{0} / \sigma\right)+\lambda_{0} i n f_{0}+\lambda_{1}\right) L f_{I P T, h=3.2, d=1} \\
& +\lambda_{1} L f_{h=3.2, d=1}+\kappa\left(\chi_{3} L f_{h=3.1, d=1}\right. \\
& -\left(S_{I P T, h=3.2, d}+L s_{I P T, h=3.2, d}\right) \\
f_{I P T, h=3.2, d=1} &
\end{aligned}
$$


Recently-Infected Latent, Post-IPT Compartments:

$$
\begin{aligned}
L f_{I P T 2, h=1.2, d=0}^{\prime}= & \left\langle f_{I P T, h=1.2, d=0}+\eta_{3} L f_{I P T 2, h=2.2, d=0}\right. \\
& -\left(\mu+\theta_{a} \mu_{h 1}+\theta_{a} \theta_{i}^{\prime} \pi_{h 1}+\sigma+\psi_{1} \lambda\right) L f_{I P T 2, h=1.2, d=0} \\
L f_{I P T 2, h=1.2, d=1}^{\prime}= & \gamma L f_{I P T, h=1.2, d=1}+\eta_{3} L f_{I P T 2, h=2.2, d=1} \\
& -\left(\mu+\theta_{a} \mu_{h 1}+\theta_{a} \pi_{h 1}+\sigma+\psi_{1} \lambda\right) L f_{I P T 2, h=1.2, d=1} \\
L f_{I P T 2, h=2.2, d=0}^{\prime}= & \gamma L f_{I P T, h=2.2, d=0} \\
& -\left(\mu+\theta_{a} \mu_{h 2}+\theta_{a} \theta_{i}^{\prime} \pi_{h 2}+\sigma+\psi_{2} \lambda\right) L f_{I P T 2, h=3.2, d=0} \\
L f_{I P T 2, h=2.2, d=1}^{\prime}= & \gamma L f_{I P T, h=2.2, d=1} \\
& -\left(\mu+\theta_{a} \mu_{h 2}+\theta_{a} \pi_{h 2}+\sigma+\psi_{2} \lambda\right) L f_{I P T 2, h=2.2, d=1} \\
L f_{I P T 2, h=3.2, d=0}^{\prime}= & \gamma f_{I P T, h=3.2, d=0} \\
& -\left(\mu+\theta_{a} \mu_{h 3}+\theta_{a} \theta_{i}^{\prime} \pi_{h 3}+\sigma+\lambda\right) L f_{I P T 2, h=3.2, d=0} \\
L f_{I P T 2, h=3.2, d=1}^{\prime}= & \gamma L f_{I P T, h=3.2, d=1} \\
& -\left(\mu+\theta_{a} \mu_{h 3}+\theta_{a} \pi_{h 3}+\sigma+\lambda\right) L f_{I P T 2, h=3.2, d=1}
\end{aligned}
$$

\section{Remotely-Infected Compartments (Ls):}

Not on IPT:

$$
\begin{aligned}
L s_{h=0, d=D}^{\prime}= & \sigma \exp \left(-\psi_{0} \lambda_{D} / \sigma\right) L f_{h=0, d=D}+\tau_{d D h 0} A_{h=0, d=D}-\left(\psi_{H} \lambda_{D}+\mu+\eta_{0}+\epsilon_{0}\right) L s_{h=0, d=D}+\rho \sum \text { deaths } \\
L s_{h=H .1, d=D}^{\prime}= & \sigma \exp \left(-\psi_{H} \lambda_{D} / \sigma\right) L f_{h=H .1, d=D}+\tau_{d D h H}\left(l t f u_{a r t T B}\right) A_{h=H .1, d=D}+\eta_{H-1} L s_{h=(H-1) .1, d=D} \\
& -\left(\psi_{H} \lambda_{D}+\eta_{H \mid H \in(1,2)}+\chi_{H}+\mu+\mu_{h H}+\epsilon_{h H}\right) L s_{h=H .1, d=D} \\
L s_{h=H .2, d=D}^{\prime}= & \exp \left(-\psi_{H} \lambda_{D} / \sigma\right) L f_{h=H .2, d=D}+\tau_{d D h H}\left(A_{h=H .2, d=D}\right. \\
& \left.+\left(1-l t f u_{a r t T B}\right)(1-\kappa) A_{h=H .1, d=D}\right)+(1-\kappa)\left(\chi_{H} L s_{h=H .1, d=D}\right) \\
& +\operatorname{detected}\left(\chi_{H} A_{h=H .1, d=D}\right)+\eta_{(H+2) \mid H \in(1,2)} L s_{h=(H+1) .2, d=D} \\
& -\left(\psi_{H} \lambda_{D}+\mu+\theta_{a} \mu_{h H}+\eta_{(H+1) \mid H \in(2,3)}+\theta_{a} \epsilon_{h H}+\kappa_{a}\right) L s_{h=H .2, d=D}
\end{aligned}
$$

Remotely-Infected Latent, on-IPT Compartments:

$$
\begin{aligned}
L s_{I P T, h=1.2, d=0}^{\prime}= & \kappa_{a} L s_{h=1.2, d=0}+\kappa\left(\chi_{1} L s_{h=1.1, d=0}+\left(1-l t f u_{\text {art } B}\right) A_{h=1.1, d=0}\right) \\
& +\eta_{3} L s_{I P T, h=2.2, d=0}+\sigma \exp \left(-\psi_{1} \lambda_{0} \inf _{0} / \sigma\right) L f_{I P T, h=1.2, d=0} \\
& -\left(\gamma+\mu+\theta_{a} \mu_{h 1}+\theta_{a} \theta_{i} \epsilon_{h 1}+\psi_{1} \lambda_{0} i n f_{0}+\psi_{1} \lambda_{1}\right) L s_{I P T, h=1.2, d=0} \\
L s_{I P T, h=1.2, d=1}^{\prime}= & \kappa_{a} L s_{h=1.2, d=1}+\kappa\left(\chi_{1} L s_{h=1.1, d=1}+\left(1-l t f u_{a r t T B}\right) A_{h=1.1, d=1}\right)+ \\
& +\eta_{3} L s_{I P T, h=2.2, d=1}+\sigma \exp \left(-\psi_{1} \lambda_{1} / \sigma\right) L f_{I P T, h=1.2, d=1} \\
& -\left(\gamma+\mu+\theta_{a} \mu_{h 1}+\theta_{a} \epsilon_{h 1}+\psi_{1} \lambda_{0} i n f_{0}+\psi_{1} \lambda_{1}\right) L s_{I P T, h=1.2, d=1} \\
= & \kappa_{a} L s_{h=2.2, d=0}+\kappa\left(\chi_{2} L s_{h=2.1, d=0}+\left(1-l t f u_{a r t T B}\right) A_{h=2.1, d=0}\right) \\
& +\eta_{4} L s_{I P T, h=3.2, d=0}+\sigma \exp \left(-\psi_{2} \lambda_{0} \inf _{0} / \sigma\right) L f_{I P T, h=2.2, d=0} \\
& -\left(\gamma+\mu+\theta_{a} \mu_{h 2}+\eta_{3}+\theta_{a} \theta_{i} \epsilon_{h 2}+\psi_{2} \lambda_{0} i n f_{0}+\psi_{2} \lambda_{1}\right) L f_{I P T, h=2.2, d=0} \\
= & \kappa_{a} L s_{h=2.2, d=1}+\kappa\left(\chi_{2} L s_{h=2.1, d=1}+\left(1-l t f u_{a r t T B}\right) A_{h=2.1, d=1}\right) \\
& +\eta_{4} L s_{I P T, h=3.2, d=1}+\sigma \exp \left(-\psi_{2} \lambda_{1} / \sigma\right) L f_{I P T, h=2.2, d=1} \\
& -\left(\gamma+\mu+\theta_{a} \mu_{h 2}+\eta_{3}+\theta_{a} \epsilon_{h 2}+\psi_{2} \lambda_{0} i n f_{0}+\psi_{2} \lambda_{1}\right) L s_{I P T, h=2.2, d=1} \\
L s_{I P T, h=2.2, d=1}^{\prime}= & \kappa_{a} L s_{h=3.2, d=0}+\kappa\left(\chi_{3} L s_{h=3.1, d=0}+\left(1-l t f u_{a r t T B}\right) A_{h=3.1, d=0}\right) \\
& +\sigma \exp \left(-\lambda_{0} \inf _{0} / \sigma\right) L f_{I P T, h=3.2, d=0} \\
& -\left(\gamma+\mu+\theta_{a} \mu_{h 3}+\eta_{4}+\theta_{a} \theta_{i} \epsilon_{h 3}+\lambda_{0} i n f_{0}+\lambda_{1}\right) L f_{I P T, h=3.2, d=0} \\
L s_{I P T, h=3.2, d=0}^{\prime} & \left(\gamma s_{h=3.2, d=1}+\kappa\left(\chi_{3} L s_{h=3.1, d=1}+\left(1-l t f u_{a r t T B}\right) A_{h=3.1, d=1}\right)\right. \\
& +\sigma \exp \left(-\lambda_{1} / \sigma\right) L f_{I P T, h=3.2, d=1} \\
& -\left(\gamma+\mu+\theta_{a} \mu_{h 3}+\eta_{4}+\theta_{a} \epsilon_{h 3}+\lambda_{0} i n f_{0}+\lambda_{1}\right) L s_{I P T, h=3.2, d=1} \\
L s_{I P T, h=3.2, d=1}^{\prime}= &
\end{aligned}
$$


Remotely-Infected Latent, Post-IPT Compartments:

$$
\begin{aligned}
L s_{I P T 2, h=1.2, d=0}^{\prime}= & \gamma L s_{I P T, h=1.2, d=0}+\eta_{3} L s_{I P T 2, h=2.2, d=0}+\sigma L s_{I P T 2, h=1.2, d=0} \\
& -\left(\mu+\theta_{a} \mu_{h 1}+\theta_{a} \theta_{i}^{\prime} \epsilon_{h 1}+\psi_{1} \lambda\right) L s_{I P T 2, h=1.2, d=0} \\
L s_{I P T 2, h=1.2, d=1}^{\prime}= & \gamma L s_{I P T, h=1.2, d=1}+\eta_{3} L s_{I P T 2, h=2.2, d=1}+\sigma L s_{I P T 2, h=1.2, d=1} \\
& -\left(\mu+\theta_{a} \mu_{h 1}+\theta_{a} \epsilon_{h 1}+\psi_{1} \lambda\right) L s_{I P T 2, h=1.2, d=1} \\
L s_{I P T 2, h=2.2, d=0}^{\prime}= & \gamma L s_{I P T, h=2.2, d=0}+\eta_{4} L s_{I P T 2, h=3.2, d=0}+\sigma L s_{I P T 2, h=2.2, d=0} \\
& -\left(\mu+\theta_{a} \mu_{h 2}+\eta_{3}+\theta_{a} \theta_{i}^{\prime} \epsilon_{h 2}+\psi_{2} \lambda\right) L s_{I P T 2, h=2.2, d=0} \\
L s_{I P T 2, h=2.2, d=1}^{\prime}= & \gamma L s_{I P T, h=2.2, d=1}+\eta_{4} L s_{I P T 2, h=3.2, d=1}+\sigma L s_{I P T 2, h=2.2, d=1} \\
& -\left(\mu+\theta_{a} \mu_{h 2}+\eta_{3}+\theta_{a} \epsilon_{h 2}+\psi_{2} \lambda\right) L s_{I P T 2, h=2.2, d=1} \\
L s_{I P T 2, h=3.2, d=0}^{\prime}= & \gamma L s_{I P T, h=3.2, d=0}+\sigma L s_{I P T 2, h=3.2, d=0} \\
& -\left(\mu+\theta_{a} \mu_{h 3}+\eta_{4}+\theta_{a} \theta_{i}^{\prime} \epsilon_{h 3}+\lambda\right) L s_{I P T 2, h=3.2, d=0} \\
L s_{I P T 2, h=3.2, d=1}^{\prime}= & \gamma L s_{I P T, h=3.2, d=1}+\sigma L s_{I P T 2, h=3.2, d=1} \\
& -\left(\mu+\theta_{a} \mu_{h 3}+\eta_{4}+\theta_{a} \epsilon_{h 3}+\lambda\right) L s_{I P T 2, h=3.2, d=1}
\end{aligned}
$$

\section{Active TB Compartments $(A)$ :}

$$
\begin{aligned}
& A_{h=0, d=0}^{\prime}=L f_{h=0, d=0} \pi_{0}+L s_{h=0, d=0} \epsilon_{0}-\left(\tau_{d 0 h 0}+\eta_{0}+\mu+\mu_{t b, h 0}\right) A_{h=0, d=0} \\
& A_{h=0, d=1}^{\prime}=L f_{h=0, d=1} \pi_{0}+L s_{h=0, d=1} \epsilon_{0}-\left(\tau_{d 1}+\eta_{0}+\mu+\mu_{t b, h 0}\right) A_{h=0, d=1} \\
& A_{h=1.1, d=0}^{\prime}=L f_{h=1.1, d=0} \pi_{h 1}+L s_{h=1.1, d=0} \epsilon_{h 1}+\eta_{0} A_{h=0, d=0}-\left(\eta_{1}+\chi_{1}+\tau_{d 0 h 1}+\mu+\theta_{h 1} \mu_{t b, h 1}+\mu_{h 1}\right) A_{h=1.1, d=0} \\
& A_{h=1.1, d=1}^{\prime}=L f_{h=1.1, d=1} \pi_{h 1}+L s_{h=1.1, d=1} \epsilon_{h 1}+\eta_{0} A_{h=0, d=1}-\left(\eta_{1}+\chi_{1}+\tau_{d 1}+\mu+\theta_{h 1} \mu_{t b, h 1}+\mu_{h 1}\right) A_{h=1.1, d=1} \\
& A_{h=2.1, d=0}^{\prime}=L f_{h=2.1, d=0} \pi_{h 2}+L s_{h=2.1, d=0} \epsilon_{h 2}+\eta_{1} A_{h=1.1, d=0}-\left(\eta_{2}+\chi_{2}+\tau_{d 0 h 2}+\mu+\theta_{h 2} \mu_{t b, h 2}+\mu_{h 2}\right) A_{h=2.1, d=0} \\
& A_{h=2.1, d=1}^{\prime}=L f_{h=2.1, d=1} \pi_{h 2}+L s_{h=2.1, d=1} \epsilon_{h 2}+\eta_{1} A_{h=1.1, d=1}-\left(\eta_{2}+\chi_{2}+\tau_{d 1}+\mu+\theta_{h 2} \mu_{t b, h 2}+\mu_{h 2}\right) A_{h=2.1, d=1} \\
& A_{h=3.1, d=0}^{\prime}=L f_{h=3.1, d=0} \pi_{h 3}+L s_{h=3.1, d=0} \epsilon_{h 3}+\eta_{2} A_{h=2.1, d=0}-\left(\chi_{3}+\tau_{d 0 h 3}+\mu+\mu_{t b, h 3}+\mu_{h 3}\right) A_{h=3.1, d=0} \\
& A_{h=3.1, d=1}^{\prime}=L f_{h=3.1, d=1} \pi_{h 3}+L s_{h=3.1, d=1} \epsilon_{h 3}+\eta_{2} A_{h=2.1, d=1}-\left(\chi_{3}+\tau_{d 1}+\mu+\mu_{t b, h 3}+\mu_{h 3}\right) A_{h=3.1, d=1} \\
& A_{h=1.2, d=0}^{\prime}=\left(L f_{h=1.2, d=0}+\theta_{i} L f_{I P T h=1.2, d=0}+\theta_{i}^{\prime} L f_{I P T 2 h=1.2, d=0}\right) \theta_{a} \pi_{h 1} \\
& +\left(L s_{h=1.2, d=0}+\theta_{i} L s_{I P T h=1.2, d=0}+\theta_{i}^{\prime} L s_{I P T 2 h=1.2, d=0}\right) \theta_{a} \epsilon_{h 1} \\
& +(1 \text { - detected }) \chi_{1} A_{h=1.1, d=0}+\eta_{3} A_{h=2.2, d=0}-\left(\tau_{d 0 h 1}+\mu+\mu_{t b h 1}+\theta_{a} \mu_{h 1}\right) A_{h=1.2, d=0} \\
& A_{h=1.2, d=1}^{\prime}=\left(L f_{h=1.2, d=1}+\theta_{i} L f_{I P T h=1.2, d=1}+\theta_{i}^{\prime} L f_{I P T 2 h=1.2, d=1}\right) \theta_{a} \pi_{h 1} \\
& +\left(L s_{h=1.2, d=1}+\theta_{i} L s_{I P T h=1.2, d=1}+\theta_{i}^{\prime} L s_{I P T 2} h=1.2, d=1\right) \theta_{a} \epsilon_{h 1} \\
& +(1 \text { - detected }) \chi_{1} A_{h=1.1, d=1}+\eta_{3} A_{h=2.2, d=1}-\left(\tau_{d 1}+\mu+\mu_{t b h 1}+\theta_{a} \mu_{h 1}\right) A_{h=1.2, d=1} \\
& A_{h=2.2, d=0}^{\prime}=\left(L f_{h=2.2, d=0}+\theta_{i} L f_{I P T h=2.2, d=0}+\theta_{i}^{\prime} L f_{I P T 2 h=2.2, d=0}\right) \theta_{a} \pi_{h 2} \\
& +\left(L s_{h=2.2, d=0}+\theta_{i} L s_{I P T h=2.2, d=0}+\theta_{i}^{\prime} L s_{I P T 2 h=2.2, d=0}\right) \theta_{a} \epsilon_{h 2} \\
& +(1 \text { - detected }) \chi_{2} A_{h=2.1, d=0}+\eta_{4} A_{h=3.2, d=0}-\left(\tau_{d 0 h 2}+\eta_{3}+\mu+\mu_{t b h 2}+\theta_{a} \mu_{h 2}\right) A_{h=2.2, d=0} \\
& A_{h=2.2, d=1}^{\prime}=\left(L f_{h=2.2, d=1}+\theta_{i} L f_{I P T h=2.2, d=1}+\theta_{i}^{\prime} L f_{I P T 2 h=2.2, d=1}\right) \theta_{a} \pi_{h 2} \\
& +\left(L s_{h=2.2, d=1}+\theta_{i} L s_{I P T h=2.2, d=1}+\theta_{i}^{\prime} L s_{I P T 2 h=2.2, d=1}\right) \theta_{a} \epsilon_{h 2} \\
& +(1 \text { - detected }) \chi_{2} A_{h=2.1, d=1}+\eta_{4} A_{h=3.2, d=1}-\left(\tau_{d 1}+\eta_{3}+\mu+\mu_{t b h 2}+\theta_{a} \mu_{h 2}\right) A_{h=2.2, d=1} \\
& A_{h=3.2, d=0}^{\prime}=\left(L f_{h=3.2, d=0}+\theta_{i} L f_{I P T h=3.2, d=0}+\theta_{i}^{\prime} L f_{I P T 2 h=3.2, d=0}\right) \theta_{a} \pi_{h 3} \\
& +\left(L s_{h=3.2, d=0}+\theta_{i} L s_{I P T h=3.2, d=0}+\theta_{i}^{\prime} L s_{I P T 2 h=3.2, d=0}\right) \theta_{a} \epsilon_{h 3} \\
& +(1 \text { - detected }) \chi_{3} A_{h=3.1, d=0}-\left(\tau_{d 0 h 3}+\eta_{4}+\mu+\mu_{t b h 3}+\theta_{a} \mu_{h 3}\right) A_{h=3.2, d=0} \\
& A_{h=3.2, d=1}^{\prime}=\left(L f_{h=3.2, d=1}+\theta_{i} L f_{I P T h=3.2, d=1}+\theta_{i}^{\prime} L f_{I P T 2 h=3.2, d=1}\right) \theta_{a} \pi_{h 3} \\
& +\left(L s_{h=3.2, d=1}+\theta_{i} L s_{I P T h=3.2, d=1}+\theta_{i}^{\prime} L s_{I P T 2 h=3.2, d=1}\right) \theta_{a} \epsilon_{h 3} \\
& +(1 \text { - detected }) \chi_{3} A_{h=3.1, d=1}-\left(\tau_{d 1}+\eta_{3}+\mu+\mu_{t b h 3}+\theta_{a} \mu_{h 3}\right) A_{h=3.2, d=1}
\end{aligned}
$$




\section{Section S1.3 Prior estimates of input parameters}

All parameter estimates and references are shown in Table 1. Our general approach to defining prior distributions for model input parameters was to identify or calculate a point estimate and plausible range for each parameter from published literature. We defaulted to a range of $\pm 40 \%$ around each point estimate, but broadened this range for parameters with a large degree of uncertainty in the prior estimate (e.g. TB transmission coefficient, CD4-dependent rates of ART initiation), and narrowed it for parameters (e.g. mortality rate in absence of TB or HIV, sensitivity of Xpert MTB/RIF) that were well understood but not well defined by our calibration data. By default, we used symmetric beta distributions with shape parameter of 3 .

Notes on select parameters are as follows:

- Recent and remote latent infection, and associated probabilities of rapid and delayed progression:

In order to model the higher risk of TB progression shortly after infection than over the subsequent lifetime with latent infection, while allowing IPT to exert effects in both this early period and the remotely-infected period, we structured our model with an explicit period of "recent infection" which all TB-infected populations passed through. We modeled this recently-infected period as having an uncertain duration, median 2 years, with an annual reactivation rate lower than that observed during the first year after infection but lower than the annualized 5-year risk of reactivation.

- Relative risk of TB progression and of HIV mortality, HIV+ on ART versus HIV + not on ART with same CD4 cell count:

The systematic review by Suthar et al (2012) estimated a hazard ratio of 0.35 (95\% CI 0.28 to 0.44 ) for TB after starting ART, however this measured effect includes the immediate effects of ART and reduced viremia as well as the effects of further immune reconstitution that occurred during the studiess' follow up periods. We modeled this immune reconstitution over time on ART as a separate effect, in order to capture the greater effect of IPT (due a higher risk of TB) sooner after ART initiation. Therefore, we used the estimate by Suthar et al as a lower bound for the effect of ART $\{i$ at a given CD4\}.

We estimated from Lawn et al 2006 that for those who start ART with CD $4<200$, approximately $3 / 4$ of the subsequent first year on ART is spent in a higher CD4 stratum. This increase in CD4 is modeled as reducing the risk of TB in the first year on ART by approximately $(3 / 4) * 40 \%=0.3$ (i.e. relative risk of 0.7 ). Therefore, we modeled the direct effect of ART as accounting for the remainder of the $60 \%$ TB risk reduction observed in the first year on ART in the meta-analysis by Low et al (2016), i.e., a multiplicative relative risk of 0.6.

- Proportion not initiating and adhering to ART during TB treatment, of TB patients not already on ART: According to local programmatic data, the proportion of PLWH who were on ART by the end of their TB treatment, among those who had not been on ART at the time of TB diagnosis, increased gradually from $40 \%$ in 2008 to $65 \%$ in 2012.) We assumed that an additional 5-20\% did not remain adherent to ART long enough to initiate IPT.

- HIV infection rates:

In absence of local data for Khayelitsha, Khayelitsha's HIV incidence was assumed to be more similar to that of provinces with HIV prevalence similar to Khayelitsha (e.g., Kwa-Zulu Natal and Eastern Cape) rather than to the Western Cape province as a whole. These provinces' estimated HIV incidence among adults 18-65 years of age was used as a basis for the prior distribution, and the model was then fit to Khayelitsha's estimated HIV prevalence at the time of the ART-IPT trial.

- ART initiation rates:

In 2010, slightly under half of individuals initiating ART in Khayelitsha had CD4> 200 (Patten et al 2014), indicating that individuals with CD4 currently 200-500 had approximately equal probabilities of reaching CD4 of 200, and then subsequently starting ART rather than dying without ART, as they did of starting ART before they reached a CD4 of 200. Therefore, the overall ART initiation rate at CD4>200 is necessarily less than the rate of dropping to CD4<200, and the ART initiate rate in absence of an active-TB diagnosis is lower still. Our prior distribution covered a wide range below this rate of HIV disease progression. In addition, because the number of estimated AIDS deaths was similar to the number of ART initiations in South Africa during this period (Johnson et al 2012; UNAIDS), we used the total mortality rate for CD $4<200$ (including baseline, HIV, and possible TB mortality) as a starting point for ART initiation rate at CD4 $<200$, again with a wide sampling range due to the considerable uncertainty in these estimates.

- Immune reconstitution on ART:

In Lawn et al (2006), among the 90\% of HIV patients who started ART with CD4 <200, 79/89 of them, if they survived, reached CD4 $>200$ within half a year, suggesting a rate of 3-4 per year in an exponential growth model. Similarly, half reached CD4 of 500 within 3 additional years, suggesting a rate of approximately 0.25 /year of transition 
to $\mathrm{CD} 4>500$.

\section{Section S2 Details of calibration methods:}

\section{Section S2.1 Calibration targets for baseline epidemic}

Each simulation (corresponding to a unique set of randomly-drawn model parameters) was seeded with one DS and one MDR active TB case in an otherwise TB- and HIV-naive population, then allowed to run to equilibrium over approximately 300 years. The following measures were then calculated:

- TB notifications: Transitions from active TB to remote latent infection (as a result of treatment).

- TB-associated mortality: All deaths occurring in individuals with active TB

- MDR prevalence among notified TB cases: Fraction of all cures of active TB that occur in MDR-TB

- HIV prevalence in the population: Proportion of entire modeled (age $>15$ ) population that is infected with HIV (with any CD4 and ART status)

- Proportion of ART initiators with CD4 count <200: Of all individuals initiating and adhering to ART, the proportion in a $\mathrm{CD} 4<200$ (advanced HIV) compartment at the time of ART initiation

- Active TB prevalence in IPT initiators: In the trial to which this was calibrated, $28 \%$ of those starting IPT were newly initiating ART, while the remaining $72 \%$ were already taking ART prior to initiating IPT. Therefore, this calibration target was calculated as a weighted average of the TB prevalence among those newly initiating ART for reasons other than a known TB diagnosis ( 0.28 weight) and the TB prevalence among those already on ART with any CD4 count (0.72 weight).

A total of 120,000 such simulations were run to generate 120,000 different TB-HIV epidemics, each with a unique set of randomly-drawn parameters. For each such simulation, each of the above-listed outputs was compared to a log-normal (for targets bounded below at zero only) or logit-normal (for proportions) probability distribution with median value and (where available) variance drawn from published data as listed in Table 1. A log of the probability density corresponding to that output was determined, and then the mean of these six log densities was calculated to generate one component of the final pseudo log-likelihood. Thus, the pseudo-likelihood associated with the fit of a given parameter set to the baseline data represented a product of the probabilities of these six model outputs, given our estimates of the corresponding epidemiologic data and their attendant uncertainty.

\section{Section S2.2 Clinical trial simulation to calibrate IPT efficacy against TB progression}

Effects of IPT on progression from latent to active TB were parametrized as (a) a reduction in risk of progression during IPT, compared to a population in the same TB, HIV, and ART state that was not receiving IPT, and (b) the proportion of this risk reduction that persisted (indefinitely) after IPT completion.

To simulate the clinical trial to produce the IPT-effect component of the pseudo-likelihood, we nested 50 simulated trials of the 12-month IPT regimen, with different randomly-chosen parameters for the protective effective of IPT, within each simulated TB epidemic. For each of the 120,000 sets of parameter values and corresponding equilibrium epidemics, 50 independent pairs of values of the parameters $\theta_{i}$ (representing the relative risk of TB progression in IPT recipients compared to non-recipients during IPT) and $k_{\theta_{i}}$ (representing how much of the risk reduction $1-\theta_{i}$ persisted after IPT completion, such that $\theta_{i}^{\prime}=1-\left(1-\theta_{i}\right) k_{\theta_{i}}$ is the relative risk of proression after IPT compared to never having received IPT) were drawn from uniform $[0,1]$ distributions using latin hypercube sampling. A nested clinical trial simulation was run for each pair of sampled value of $\theta_{i}$ and $k_{\theta_{i}}$. This simulated clinical trial was set up as follows: From the population at equilibrium, a representative sample of 622 individuals on ART (corresponding to the treatment group size in the trial by Rangaka et al., Lancet 2014:384:682) was created (proportional to the distribution of the population across ART states), and all were immediately started on 12 months of IPT. Parallel simulations were also run in which no IPT was provided to these 622 individuals. These groups were followed for 2.5 years after IPT introduction (the median follow up in the clinical trial), tracking incident TB cases as well as total time at risk, during treatment or placebo and over the entire follow-up period, in each group.

The resulting incidence rate ratios, comparing IPT-recipients to non-recipients during treatment or placebo and over the entire 2.5-year period, were compared to log-normal distributions corresponding to the hazard ratio observed by Rangaka et al: 0.63 (95\% CI 0.41-0.94) overall (mean $\log =\log (0.63)$ and $s d \log =\log (1.22)$ ), and $0.52(0.27-1.01)$ during treatment 
(mean $\log =0.52$, sd $\log =1.4$ ); a log probability density was calculated for each incidence rate ratio and corresponding log-normal target distribution. The mean of these two log-normal densities was added to the first pseudo log-likelihood term described in the paragraph above (representing the fit of the model prior to introducing IPT) to yield the final log pseudo-likelihood.

\section{Section S2.3 Parameter resampling based on pseudo-likelihood}

Simulations (all 120,000*50 pairs of underlying epidemic simulation combined with randomly-drawn $\theta_{i}$ and $k_{\theta_{i}}$ values) were then re-sampled according to the exponentiated pseudo-likelihood value. Thus, the weight given to each set of parameter values in determining model results corresponded to the combined fit of the simulation to the observed TB-HIV epidemic in Khayelitsha and the observed effects of IPT in a clinical trial.

\section{Section S3 Modeled Interventions and sensitivity analysis scenarios}

\section{Baseline model - no intervention:}

- $\kappa=0$ (no IPT initiation for those initiating ART)

- $\kappa_{a}=0$ (no IPT initiation for those already on ART)

Primary intervention (12-month IPT, for ART initiators and some already on ART):

- $\kappa=0.85$ ( $85 \%$ of ART initiators without active TB also receive IPT (accounting for $15 \%$ nonadherence))

- $\kappa_{a}=0.15$ (each year, $15 \%$ of those already on ART but not IPT are initiated on IPT)

- $\gamma=1$ (12-month duration of IPT regimen)

- $\theta_{i}$ and $\theta_{i}^{\prime}$ fit to trial data as described above

- $i n f_{0}=0$ (active IPT protects against new infection with drug-susceptible TB strains)

\section{Continuous-IPT intervention}

- $\kappa=0.85$

- $\kappa_{a}=0.15$

- $\gamma=0$ (IPT does not end once started)

- $\theta_{i}$ and $\theta_{i}^{\prime}$ fit to trial data as described above

- $i n f_{0}=0$

\section{2-month IPT limited to ART initiators}

- $\kappa=0.85$

- $\kappa_{a}=0$ (no IPT initiation for those already on ART before start of IPT intervention)

- $\gamma=1(12-$ month IPT)

- $\theta_{i}$ and $\theta_{i}^{\prime}$ fit to trial data as described above

- $i n f_{0}=0$

No-IPT-effect sensitivity analysis (to identify in which populations the cases averted by IPT would occur)

- $\kappa=0.85$

- $\kappa_{a}=0.15$

- $\gamma=1$ (12-month IPT)

- $\theta_{i}=1$ (no reduction in risk of TB progression during or after IPT)

- $i n f_{0}=1$ (no protection against TB infection while taking IPT)

\section{Increasing ART coverage scenario}

For $t \geq 0$ : 
- $\chi_{1}(t)=\chi_{2}=\chi_{2(t=0)} *(1+0.5 *(t))$

- $\chi_{3}(t)=\chi_{3(t=0)} *(1+0.1 *(t))$

- $\eta_{0}(t)=\eta_{0(t=0)} * \frac{\text { prevalence of untreated HIV at time } t}{\text { prevalence of untreated HIV at } \mathrm{t}=0}$

Lower-incidence scenario (sensitivity analysis)

- TB notification calibration target (midpoint and both endpoints) reduced $67 \%$

- TB mortality calibration target (midpoint and both endpoints) reduced $67 \%$

- MDR prevalence (among active TB initiating effective treatment) calibration target unchanged

- HIV prevalence calibration target unchanged

- CD4 (at ART initiation) calibration target unchanged

- target for TB prevalence among ART initiators reduced 67\%

- Prior distributions scaled by 0.33 for $\beta, 0.67$ for $\pi_{0}, \pi_{h}, \epsilon_{0}$, and $\epsilon_{h}$.

- Resulting size of calibrated TB epidemic: TB incidence 852/100,000/year (95\% CrI 740-983), TB notification rate 593/100,000/year (95\% CrI 510-699), TB prevalence 893/100,000 (95\% CrI 736-1072).

\section{Section S4 Supplementary Results}

\section{Section S4.1 Model fit to calibration targets:}

From an original $120,000 * 50$ parameter sets, 100,000 parameter sets were resampled with replacement, with weighting based on their pseudo-likelihood function values. This resulted in 9,746 unique simulations in the posterior, representing 1,365 unique underlying TB-HIV epidemics (with additional variability in the estimate of the IPT efficacy parameter).

Epidemiologic measures in the calibrated model corresponded closely to their target distributions (Supplementary Figure S2).

The prior and post-calibration values of input parameters to the baseline model are shown in Supplementary Figure S1.

\section{Section S4.2 Other epidemiologic and IPT-efficacy outputs}

Additional epidemiological outputs not included in the pseudo-likelihood or used for calibration were also consistent with available provincial and city data:

- TB incidence: 2408 (95\% CrI 2170-2622) per 100,000 per year

- TB prevalence: 2865 (95\% CrI 2334-3465) per 100,000

- HIV prevalence among cases of active TB: 79\% (95\% CrI 68-88\%)

- LTBI prevalence among PLWH: 96\% (95\% CrI 90-98\%)

- ART coverage among PLWH: 48\% (95\% CrI 40-54\%)

After using the hazard ratio of incident TB in a simulated clinical trial to calibrate the estimates of IPT efficacy parameters, we estimated relative rates of TB progression, compared to never having received IPT, to be $\theta_{i}=0.54(95 \% \mathrm{CrI} 0.38-0.70)$ during IPT, and $\theta_{i}^{\prime}=0.76$ (95\% CrI 0.57-0.97) after IPT. The estimated proportion of IPT effect that persisted after the end of IPT $\left(k_{\theta_{i}}\right)$ was imprecisely estimated by the trial data (median $0.54,95 \%$ CrI 0.05-0.97), and its estimated value was correlated (Pearson $\mathrm{R}=0.44$ ) with that of $\theta_{i}$.

Of all individuals with HIV, most (77\%, 95\% CrI 71-84\%) were modeled as being in the highest-CD4-count compartments (CD4>500): half of them (51\% of those in a CD4>500 compartment, 95\% CrI 41-61\%) because their HIV had not yet progressed to a more advanced state, and half of them because they had experienced immune reconstitution on ART. Another 18\% (13-24\%) had a CD4 in the 200-500 range, and 4\% (3-6\%) of all PLWH had a CD4<200 at any given time. Of individuals initiating and adhering to ART, 67\% (56-75\%) initiated at CD4-dependent rates, with the remainder initiating ART due to a diagnosis of active TB. 

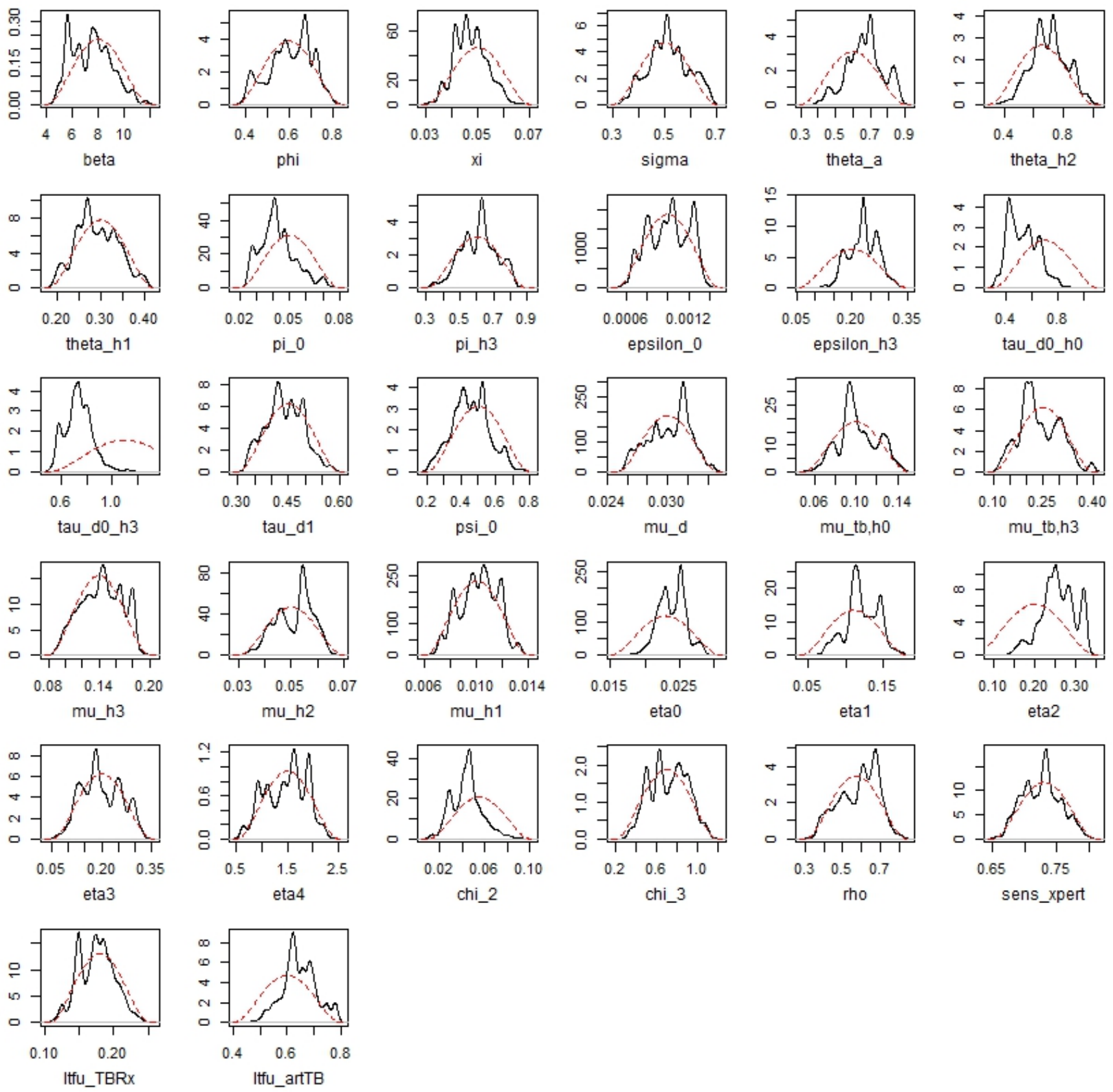

Figure S1: Prior and posterior distributions of model input parameters. 

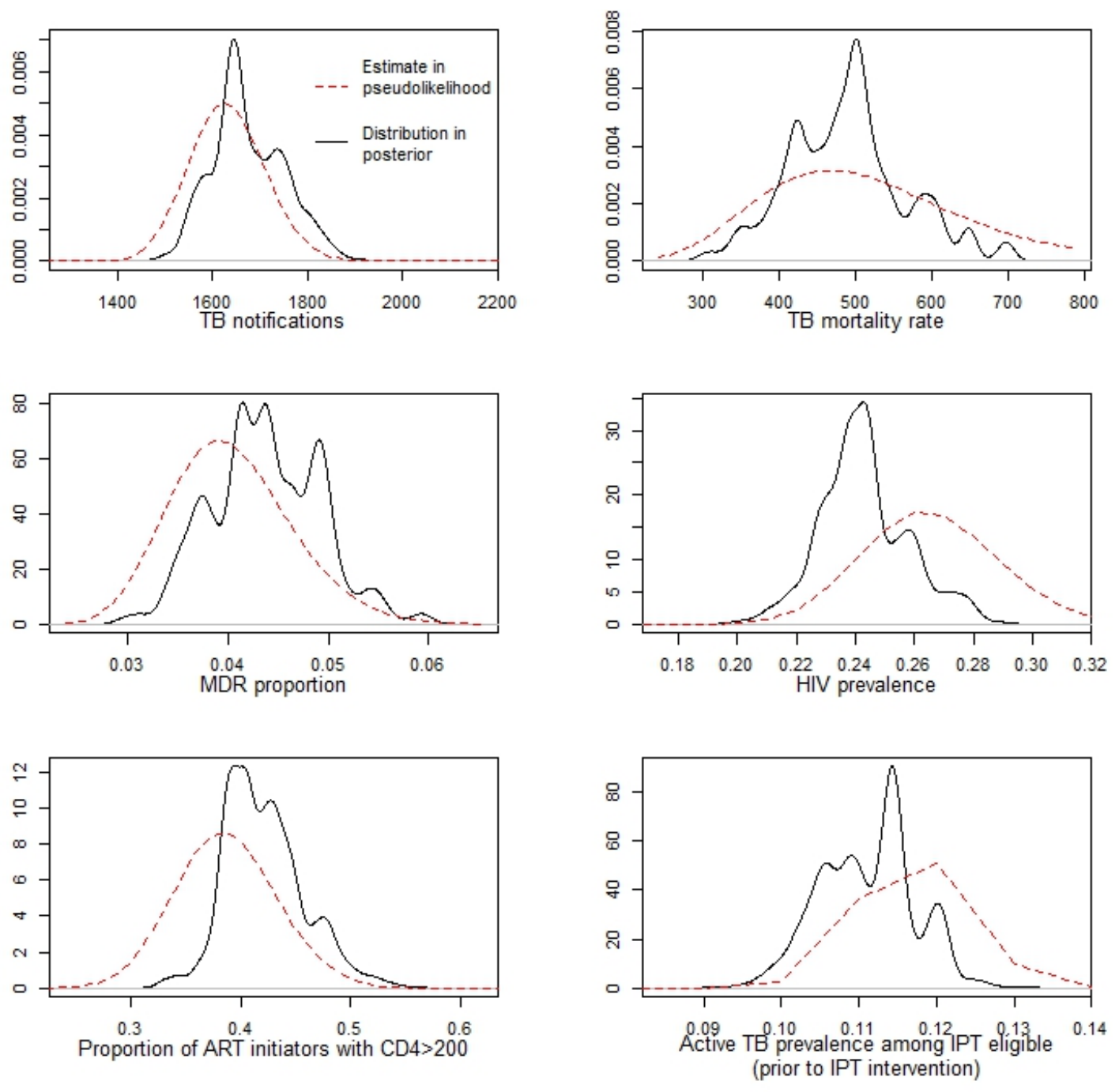

Figure S2: Model fit to calibration targets. 

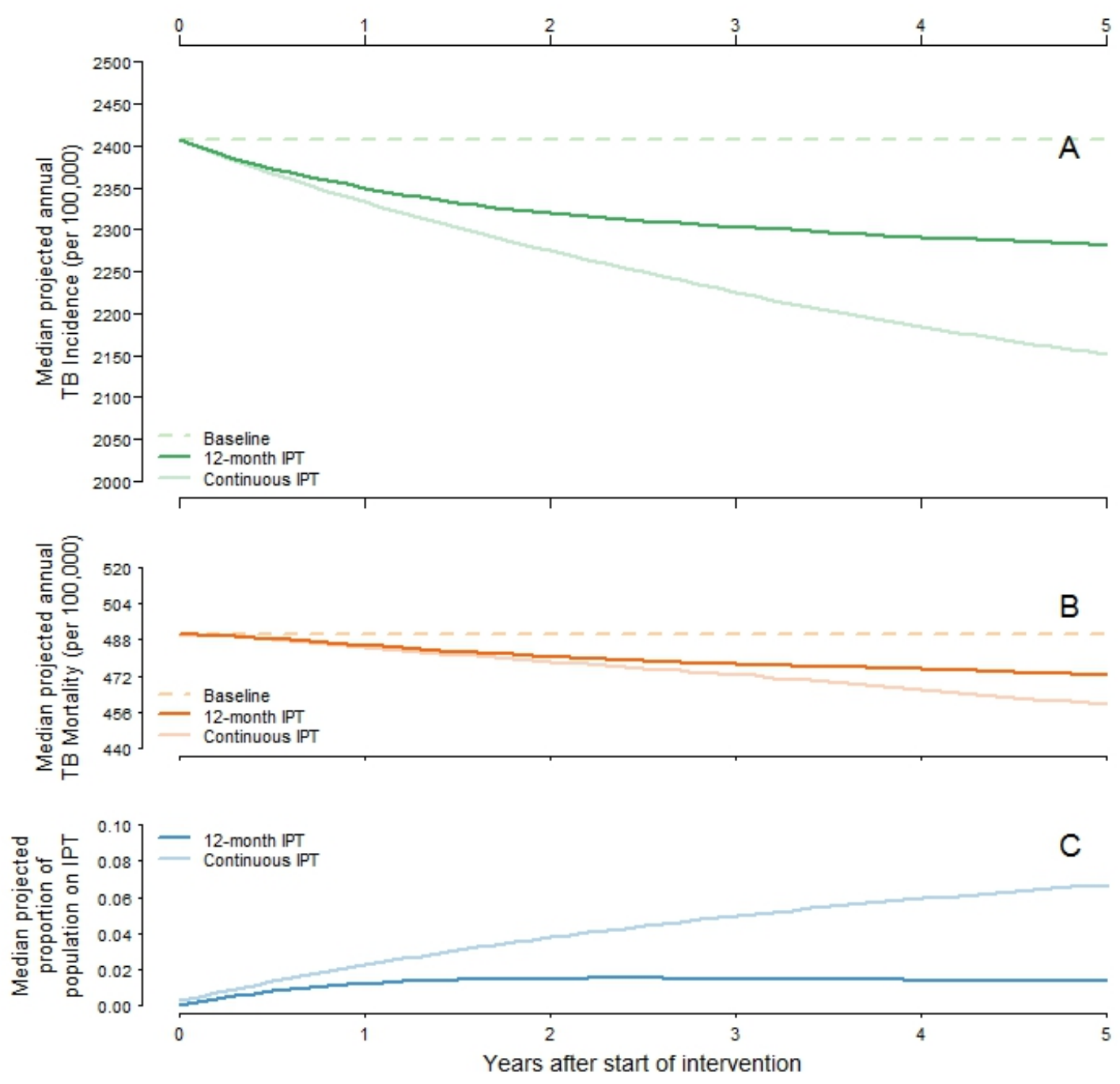

Figure S3: Population Level Impact of IPT comparing the median projected incidence, mortality, and IPT coverage for a continuous regimen (darker solid lines) to a 12-month regimen (lighter solid lines). Panels A and B illustrate the median projected incidence (A, green) and mortality (B, orange) in both the baseline (dashed line) and the intervention scenarios (solid line). Panel C (bottom) illustrates the median proportion of the population at any one time actively on IPT within Khayelitsha after the start of the intervention (time 0). Ninety-five percent credible intervals are omitted for clarity but reported in the text.

\section{Section S4.3 Additional results with continuous IPT regimen}

Five-year outcomes with 12-month and continuous IPT regimens are compared graphically in Supplementary Figure S3.

Additionally, we estimated that an additional 46 (95\% CrI 24-117) person-years of continuous IPT were required to prevent an additional TB case not prevented by 12 -month IPT.

\section{Section S4.4 Additional sensitivity analysis: IPT prevents infection only}

Finally, to distinguish effects on reinfection versus progression of preexisting latent infection among those treated, we considered a sensitivity analysis in which the only impact of IPT was to prevent new infection $\left(\theta_{i}=0\right)$, and compared the cumulative averted cases and deaths to those when all IPT effects were modeled. Although the annual risk of TB infection was high in the setting modeled, we found that prevention of new TB infection during IPT accounted for only 16\% (95\% CrI 8-23\%) of the TB cases prevented by the 12-month ART-linked IPT regimen over a 5-year period of implementation. Contributing factors to this result include the high prevalence of preexisting latent infection ( $>75 \%)$ among HIV-infected individuals, the high reactivation risk of infections acquired shortly before starting ART and IPT, and the larger amount of collective time spent by HIV-infected individuals in the post-IPT state (and vulnerable to reinfection) than on IPT when IPT was implemented as a 12-month regimen. 